\title{
Digital Imaging Shared Resource
}

National Cancer Institute

\section{Source}

National Cancer Institute. Digital Imaging Shared Resource. NCI Thesaurus. Code C39376.

The Digital Imaging Shared Resource provides Cancer Center investigators with support in high-resolution deconvolution microscopy, digital epi-fluorescence and brightfield microscopy, quantitative image analysis, cell microinjection and resulting biological analyses, and photo-production. 